\title{
Perfil de adolescentes em privação de liberdade: eventos estressores, uso de drogas e expectativas de futuro
}

Profile of adolescents from deprivation of freedom: stressful events, drug use and future expectations

Perfil de los adolescentes en privación de libertad: eventos estresores, uso de drogas y expectativas de futuro

\author{
Fernanda Ludke Nardi* \\ Guilherme Machado Jahn ${ }^{* *}$ \\ Débora Dalbosco Dell'Aglio ${ }^{* * *}$
}

\begin{abstract}
Resumo
Este estudo investigou o perfil de adolescentes em conflito com a lei que cumpriam medida socioeducativa em regime fechado nas unidades da FASE-RS, em Porto Alegre, observando a ocorrência de eventos estressores, uso de drogas e expectativas de futuro. Participaram 143 adolescentes, de 14 a 20 anos $(M=17,23 ; d p=1,19)$, que responderam a uma ficha de dados sociodemográficos e a um questionário com questões relacionadas a fatores de risco e de proteção. Os dados apontam para a predominância de adolescentes de cor branca e baixa escolaridade, a maioria morava com a mãe e irmãos, $74,8 \%$ já vivenciaram a morte de alguém importante, mais de 70\% já fizeram uso de drogas e 18,9\% pensaram em se matar. Os participantes apresentaram boas expectativas de futuro, mas baixas expectativas quanto a concluir os estudos. Destacase a importância do contexto que envolve os adolescentes em conflito com lei, assim como a necessidade de investimento em políticas públicas para essa população.
\end{abstract}

Palavras-chave: Adolescente em conflito com a lei. Drogas. Eventos estressores. Expectativas de futuro.

\section{Abstract}

This study has investigated the profile of young offenders who were deprived of freedom in the units of FASE-RS in Porto Alegre, observing

\footnotetext{
Doutora em Psicologia do Desenvolvimento pela Universidade Federal do Rio Grande do Sul. E-mail: fernanda.nardi@ yahoo.com.br.

** Monitor acadêmico da Universidade Federal do Rio Grande do Sul. E-mail: guijahn@gmail.com.

*** Docente do Programa de Pós-graduação em Psicologia da Universidade Federal do Rio Grande do Sul. E-mail: dalbosco@ cpovo.net.
} 
the occurrence of stressful events, drug use on future expectations. The participants were 143 adolescents aged between 14 to 20 years $(\mathrm{M}=17.23$; sd $=1.19)$, who have completed a socio-demographic form and a questionnaire related to risk and protection factors. The data obtained pointed to the predominance of white adolescents and low schooling. Most of whom lived with the mother and siblings, 74.8\% had already experienced the death of someone important, over $70 \%$ had already used drugs and $18.9 \%$ thought about killing themselves. The participants presented good expectations for the future, but low expectations concerning the conclusion of studies. The importance of the context that involves adolescents in conflict with the law is highlighted, well as the need for investment in public policies for this sector of the population.

Keywords: Young offenders. Drugs. Stressful events. Future expectations.

\section{Resumen}

Este estudio investigó el perfil de adolescentes en conflicto con la ley que cumplían medida socioeducativa en régimen cerrado en las unidades de la FASE-RS, en Porto Alegre, observando la ocurrencia de eventos estresores, uso de drogas y expectativas de futuro. Participaron 143 adolescentes, de 14 la 20 años $(M=17,23 ; d p=1,19)$, que respondieron a una ficha de datos socio-demográficos y a un cuestionario acerca de factores de riesgo y protección. Los datos apuntan a la predominancia de adolescentes de color blanco y baja escolaridad, la mayoría vivía con su madre y sus hermanos, el 74,8\% experimentó la muerte de alguien importante, más del $70 \%$ usó drogas y el 18,9\% pensó en suicidarse. Los participantes presentaron buenas expectativas de futuro, pero bajas expectativas cuanto a concluir los estudios. Se destaca la importancia del contexto que envuelve a los adolescentes en conflicto con la ley, así como la necesidad de inversión en políticas públicas para esa población.

Palabras clave: Adolescente en conflicto con la ley. Drogas. Eventos estresores. Expectativas de futuro.

\footnotetext{
A elaboração de políticas públicas de prevenção à infração juvenil tem sido tema de grande relevância em muitos países, tendo em vista que o ato infracional na adolescência pode acarretar consequências negativas tanto para os jovens como para seu ambiente (Hein, 2004). No Brasil, o adolescente que comete algum tipo de ato infracional fica submetido às medidas socioeducativas previstas no Estatuto da Criança e do Adolescente
} 
[ECA] Lei n. 8.069 (1990). No que concerne ao cometimento de atos infracionais, o ECA define diretrizes para a responsabilização dos jovens por meio de medidas socioeducativas, sempre que o ato infracional for cometido antes dos 18 anos. Essas medidas podem ser cumpridas pelo adolescente tanto em liberdade (como no caso da advertência, da prestação de serviços à comunidade e da liberdade assistida) como em regime de internação (por um período máximo de três anos) Lei n. 8.069 (1990).

Estudos com essa população têm retratado a exposição desses adolescentes a variados fatores de risco (Zamora, 2008). Frequentemente, esses adolescentes estão envolvidos em situações de perigo, como esportes violentos, gangues, uso e tráfico de drogas, crime violento e organizado (Adorno, 2010). Alguns autores têm debatido a questão do adolescente tanto no papel de agressor quanto de vítima, demonstrando a exposição desses jovens a fatores de risco, ao mesmo tempo em que eles mesmos se colocam em risco (Adorno, 2010; Deperon \& Pinho, 2012). Outros fatores que contribuem para gerar consequências negativas no desenvolvimento, e que frequentemente estão presentes nesses casos, são o uso de drogas, a baixa escolaridade e a vivência de distintos eventos estressores (Gallo \& Williams, 2005; Malvasi, 2011; Maruschi, Estevão \& Bazon, 2012; Pereira \& Sudbrack, 2008).

No que se refere ao uso de drogas, Vasters e Pillon (2011) mencionam que é na adolescência que o indivíduo busca, de forma mais intensa, seu universo de experimentações e identificações, geralmente com associações aos grupos de pares. $\mathrm{O}$ consumo de drogas tem atingido maior amplitude na sociedade contemporânea entre os adolescentes. De acordo com os autores, tal uso tem ocorrido precocemente. Para Ferigolo et al. (2004), quanto mais cedo ocorre o início do uso de álcool e tabaco, maior a vulnerabilidade de desenvolver o abuso e a dependência dessas duas substâncias, assim como o uso de drogas ilícitas. O consumo e o uso abusivo de drogas na adolescência podem acarretar uma série de consequências negativas em múltiplos domínios de vida, como prejuízos nas funçôes cognitivas (Rigoni, Oliveira, Moraes \& Zambom, 2007); prejuízos no desempenho de habilidades sociais e conflitos interpessoais (Wagner \& Oliveira, 2007); infração juvenil (Anderson, 1999; Nardi, Cunha, Bizarro \& Dell'Aglio, 2012; Pacheco \& Hutz, 2009; Pereira $\&$ Sudbrack, 2008), entre outros.

Em estudo de revisão, Senna e Dessen (2012) destacam que, apesar dos avanços na compreensão da adolescência, ainda se pode identificar uma tendência a caracterizar esse período como um momento no curso de vida, repleto de dificuldades, conflitos, alteraçôes constantes de humor e comportamentos de risco. Sapienza e Pedromônico (2005) ressaltavam que 
a adolescência poderia ser considerada um período de maior vulnerabilidade para o impacto de eventos estressores. Para Gröer, Thomas e Schoffner (1992), muitos eventos estressores vivenciados pelos adolescentes estão relacionados à aquisição e à transição de papéis, tais como adaptação a novos ambientes, mudanças nas relações com pares, questões de separação-individuação e de preparação para a universidade ou para o trabalho.

Uma das possíveis consequências do impacto de eventos estressores, tais como desemprego ou prisão, é a ideação ou tentativa de suicídio (Móscicki, 1997). Em estudo de revisão, Braga (2011) aponta que o suicídio na adolescência tem aumentado nas últimas décadas e tem sido considerado um problema de saúde pública mundial, que traz consequências negativas não apenas para a família da vítima, mas para o meio social no qual o adolescente está inserido. Vermeiren (2003), em estudo de revisão sobre suicídio em adolescentes autores de ato infracional, mostrou que a taxa de ideação suicida é similar entre adolescentes infratores e jovens estudantes, sendo maior a taxa no sexo feminino. Além disso, o autor também explicitou que os fatores de risco para o suicídio em jovens com comportamento antissocial incluem idade, abuso de substâncias, abuso físico e sexual, assim como depressão.

No entanto existem fatores de proteção que podem atuar na vida dos jovens em conflito com a lei, mediando os riscos e contribuindo para resultados positivos no desenvolvimento. Lodewijks, Ruiter e Doreleijers (2010) ressaltam que a maioria dos estudos sobre preditores de reincidência do ato infracional em adolescentes tem focado nos fatores de risco em vez de focar em fatores de proteção. Esses autores mencionam ainda que os fatores de proteção mais significativos no que diz respeito à desistência do comportamento antissocial são atitudes positivas em relação à escola, suporte social e vínculos saudáveis.

Os projetos de vida, que refletem aspirações e desejo de realizações para o futuro (Nascimento, 2006), assumem grande importância para os indivíduos, sobretudo na adolescência. Os projetos de vida podem ser considerados como fatores de proteção (Costa \& Assis, 2006) pois contribuem na prevenção ao ato infracional. Furlani e Bomfim (2010) defendem que uma sociedade que não fornece diretrizes sólidas e saudáveis para que os adolescentes consigam elaborar projetos de vida pode levar ao aumento dos índices de violência juvenil.

Assim, estudar fatores de proteção e de risco presentes na vida de adolescentes em cumprimento de medida em meio fechado é importante já que auxilia na criação e no aperfeiçoamento de programas de intervenção para esses jovens e suas famílias, tanto nas unidades de internação como nas 
comunidades. Portanto o objetivo deste estudo foi analisar o perfil desses adolescentes, no que concerne ao uso de drogas, suicídio, eventos estressores e expectativas para o futuro. A importância de estudar a presença desses fatores na juventude se deve ao fato de que o processo de formação do adolescente depende de diversas variáveis biológicas, psicológicas e sociais que fazem parte do contexto de vida desses indivíduos (Garbarino, 2009), as quais muitas vezes não são tratadas durante o acompanhamento do jovem em conflito com a lei.

\section{Método}

Participaram da pesquisa 143 adolescentes em conflito com a lei que cumpriam medida em regime fechado nas unidades da Fundação de Atendimento Socioeducativo Rio Grande do Sul (FASE-RS) de Porto Alegre. Os participantes dessa pesquisa tinham entre 14 e 20 anos $(M=17,23$; $\mathrm{dp}=1,19)$, sendo que $128(89,5 \%)$ eram do sexo masculino e $15(10,5 \%)$ do sexo feminino. Segundo a Assessoria de Informação e Gestão da FASERS, no período de janeiro a maio de 2010, havia 565 meninos e 31 meninas internados na instituição, demonstrando que a amostra representou respectivamente $23 \%$ dos meninos e $48 \%$ das meninas em cumprimento de medida em regime fechado durante o período da coleta de dados.

Os participantes responderam a uma ficha de dados referente às visitas de familiares, ao ato infracional cometido, ao tempo de permanência na instituição, entre outros, e a um questionário (Dell'Aglio, Koller, CerqueiraSantos \& Colaço, 2011), o qual foi reduzido para a coleta de dados na FASE, sendo composto por 47 questôes objetivas sobre fatores de risco e de proteção. O questionário englobou questões sobre educação, eventos estressores, violência intra e extrafamiliar, suicídio, uso de drogas e expectativas de futuro, entre outras.

Após a assinatura do termo de concordância da instituição, os dados foram coletados em cinco unidades de internação, sendo uma unidade feminina (a única existente). O critério de seleção dos participantes foi escolaridade mínima de $5^{\mathrm{a}}$ série e que pudessem compreender adequadamente os instrumentos. Alguns adolescentes com menor escolaridade, mas que desejaram participar, responderam individualmente ao questionário, com o auxílio dos pesquisadores. Os adolescentes assinaram o termo de assentimento, concordando em participar do estudo, e a aplicação dos instrumentos foi coletiva, em grupos de seis a oito jovens, com duração aproximada de 60 minutos. 
Foram feitas análises descritivas para identificar o perfil sociodemográfico dos jovens. Além disso, foi realizado o teste qui-quadrado para verificar diferenças entre os sexos. Foram empregadas questôes referentes ao ato infracional cometido, dados sociodemográficos, situação escolar, uso de drogas, eventos estressores, ideação e tentativa de suicídio, assim como expectativas de futuro. Essas questões foram selecionadas por englobarem, de forma geral, fatores de risco e de proteção presentes na vida desses jovens. A questão sobre as expectativas para o futuro foi baseada no instrumento construído por Günther e Günther (1998), contendo itens em escala tipo Likert com cinco opções de resposta, nos quais os sujeitos respondiam quais as chances que acreditavam ter de terminar o ensino médio, ingressar em uma universidade, entre outros.

\section{Resultados e discussão}

Os dados levantados apontaram um percentual de meninas cumprindo medida em regime fechado inferior aos meninos. Esses dados são semelhantes aos índices observados no relatório sobre a situação dos direitos da criança e do adolescente no Brasil - Associação Nacional dos Centros de Defesa da Criança e do Adolescente (ANCED) e do Fórum Nacional Permanente das Entidades Não Governamentais de Defesa dos Direitos da Criança e do Adolescente (Fórum DCA) (2004), o qual mostrou que o universo das medidas em meio fechado é predominantemente masculino, com apenas $6 \%$ dos internos do sexo feminino. Esse dado remete a uma reflexão sobre diferenças relacionadas a estereótipos de gênero. Para Fukuda, Brasil e Alves (2009), questôes culturais envolvem o comportamento dos indivíduos segundo o gênero. Garbarino (2009) também indica essa influência, apontando que crenças culturais baseadas na diferença entre os sexos, que justifiquem determinados comportamentos, contribuem para um maior envolvimento de meninos em comportamentos agressivos e externalizantes. Portanto diferenças sexuais e expectativas de gênero podem influenciar o comportamento dos adolescentes e contribuir no esclarecimento da prevalência de meninos em cumprimento de medidas socioeducativas.

Conforme a tabela 1, a maioria dos adolescentes internados na FASE era de cor branca $(60,1 \%)$. Porém a realidade brasileira é um pouco diferente, conforme relatório da ANCED (ANCED, FÓRUM DCA, 2004), com 60\% dos adolescentes em cumprimento de medida de internação de cor negra, refletindo a maior vulnerabilidade dos negros em uma sociedade em que são historicamente os mais atingidos pela desigualdade social. Portanto se percebe que os resultados quanto à cor, apontados por este estudo, podem 
estar refletindo uma característica da população da Região Sul do País. De acordo com censo realizado pelo Instituto Brasileiro de Geografia e Estatística [IBGE] (2010), as regiōes Sul e Sudeste são aquelas que concentram o maior número de brasileiros de cor branca, sendo que, no Rio Grande do Sul, 83\% da população são de cor branca (IBGE, 2010).

Quanto à escolaridade, a maioria estava entre as $5^{\mathrm{a}}$ e $7^{\mathrm{a}}$ séries, o que demonstra uma defasagem escolar quando observada a média de idade dos participantes. Ressalta-se que o critério de seleção, considerando a escolaridade, pode ter levado a um viés na amostra, sendo que a média de escolaridade dos adolescentes em conflito com a lei pode ser ainda menor do que a observada nesse estudo. Essa defasagem pode ser explicada pelo percentual de expulsão da escola $(31,5 \%)$ e, mais ainda, pela reprovação escolar $(91,6 \%)$. Esses dados revelam características que já vêm sendo apontadas por diversos outros estudos, que mostram a baixa escolaridade dos adolescentes em conflito com a lei (Davoglio \& Gauer, 2011; Gallo \& Williams, 2005; Oliveira \& Assis, 1999). Segundo relatório que avaliou a escolaridade dos adolescentes em cumprimento de medida em meio fechado no Brasil, 51\% dos jovens não frequentavam a escola quando praticaram o ato infracional e metade dos internos não tinha concluído o ensino fundamental (ANCED, FÓRUM DCA, 2004).

Tabela 1 - Características gerais dos adolescentes em cumprimento de medida em regime fechado

\begin{tabular}{l|l|l|l}
\hline & Variável & Frequência & $\%$ \\
\hline Cor & Branca & 86 & 60,1 \\
& Negra & 38 & 26,6 \\
Escolaridade* $^{*}$ & Outras & 19 & 13,3 \\
& 1a a 4a série & 7 & 4,9 \\
& 5a série & 27 & 18,9 \\
& 6a série & 35 & 24,5 \\
& 7a série & 26 & 18,2 \\
& 8a série & 16 & 11,2 \\
& Ensino médio & 27 & 18,9 \\
Morava com & Ex* & 45 & 31,5 \\
& Expulso da escola & 131 & 91,6 \\
& Raprovado na escola & 41 & 28,7 \\
& Mai & 99 & 69,2 \\
& Padrasto & 18 & 12,6 \\
& Madrasta & 4 & 2,8 \\
& Irmãos & 74 & 51,7 \\
& Avố & 9 & 6,3 \\
& Avó & 16 & 11,2 \\
& Filhos & 6 & 4,2 \\
& Companheiro(a) & 26 & 18,2 \\
\hline
\end{tabular}

Nota: * cinco participantes não informaram a escolaridade; ${ }^{* *}$ os itens não são excludentes.

Fonte: Dados da pesquisa. 
Quando observados os percentuais de membros da família que viviam junto com o adolescente, percebe-se que mais da metade morava com a mãe $(69,2 \%)$, mas muitos ainda viviam sem essa figura considerada tão importante para o desenvolvimento. Por outro lado, pode-se observar que apenas $28,7 \%$ deles moravam com o pai e $12,6 \%$ tinham a presença do padrasto, indicando que muitos deles viviam em famílias monoparentais. Alguns estudos apontam a monoparentalidade como um aspecto presente na maioria das famílias de jovens em conflito com a lei (Dias, Arpini \& Simon, 2011; Feijó \& Assis, 2004) e outros ainda destacam a ausência paterna nos lares de jovens infratores (Branco, Wagner \& Demarchi, 2008; Dell'Aglio, Santos \& Borges, 2004). Dessa forma, ocorre uma situação de desamparo nessas famílias, visto que a mãe acaba ficando sobrecarregada, uma vez que ela é a principal responsável por suprir as necessidades econômicas, sociais e afetivas dos filhos. Em revisões de estudos sobre o tema, Eizirik e Bergmann (2004) encontraram que a presença de comportamento antissocial em qualquer membro da família é mais provável quando o pai é ausente ou não participativo, o que teria potencial para gerar conflitos no desenvolvimento psicológico da criança. Porém essa influência é discutida, pois enquanto, para alguns profissionais da área, a influência da ausência do pai constitui-se um fator negativo, outros apontam para uma ausência de influência. De acordo com Eizirik e Bergmann (2004), o que se deve levar em consideração nas situações de ausência paterna no desenvolvimento da criança ou do adolescente é o papel materno. Desse relacionamento pode surgir uma maior ou menor predisposição para os conflitos associados à falta do pai, ou seja, tal relação agiria como mediadora das repercussões dessa ausência na vida emocional dos jovens.

Quanto aos atos infracionais cometidos pelos participantes deste estudo, 34,3\% haviam cometido assalto, $26,6 \%$ estavam envolvidos no tráfico de drogas e $14,7 \%$ haviam cometido homicídio. Com percentuais menores, 5,6\% dos jovens estavam cumprindo medida em regime fechado devido à tentativa de homicídio; $2,1 \%$, devido a sequestro; e $6,3 \%$ estavam envolvidos em outros atos, tais como furto, roubo e porte de arma. Percebe-se que a maioria dos adolescentes internados praticou atos que se constituem em grave ameaça ou violência contra pessoa. Esses dados estão de acordo com o relatório sobre a situação dessa população no Brasil, o qual mostrou que $29,5 \%$ haviam cometido roubo; $18,6 \%$, homicídio; $14,8 \%$, furto; e $8,7 \%$, tráfico de drogas. De acordo com o ECA (1990) Lei n. 8.069/90, somente atos infracionais reiterados 
ou praticados sob grave ameaça ou violência à pessoa constituemse hipóteses de medida de internação, ou ainda quando tiver sido descumprida, de modo reiterado e injustificável, medida anteriormente imposta. No caso de outros atos, pode-se avaliar a possibilidade de ser cumprida medida em regime aberto, satisfazendo, assim, o direito de adolescentes à convivência familiar e comunitária. Além disso, Muller, Barboza, Oliveira, Santos e Paludo (2009) ressaltam a severidade da medida de internação, pois retira o adolescente do convívio social e de sua família.

Tabela 2 - Características referentes ao uso de substâncias psicoativas

\begin{tabular}{|c|c|c|c|c|}
\hline \multirow[t]{2}{*}{ Variável } & \multicolumn{3}{|c|}{$f$ e \% } & \\
\hline & Masc. & Fem. & Total & \\
\hline $\begin{array}{l}\text { Tem familiar usuário de } \\
\text { drogas }\end{array}$ & $\begin{array}{l}67 \\
(54 \%)\end{array}$ & $\begin{array}{l}10 \\
(66,7 \%)\end{array}$ & $\begin{array}{l}77 \\
(53,8 \%)\end{array}$ & \\
\hline \multirow[t]{2}{*}{$\begin{array}{l}\text { Tem amigo usuário de } \\
\text { drogas }\end{array}$} & $\begin{array}{l}115 \\
(92,7 \%)\end{array}$ & $\begin{array}{l}12 \\
(80 \%)\end{array}$ & $\begin{array}{l}127 \\
(88,8 \%)\end{array}$ & \\
\hline & & & & Idade de início \\
\hline Usava bebida alcoólica & $\begin{array}{l}93 \\
(87,7 \%)\end{array}$ & $\begin{array}{l}11 \\
(73,3 \%)\end{array}$ & $\begin{array}{l}104 \\
(72,7 \%)\end{array}$ & $M=13,6, d p=1,85$ \\
\hline Usava cigarro & $\begin{array}{l}75 \\
(72,1 \%)\end{array}$ & $\begin{array}{l}11 \\
(73,3 \%)\end{array}$ & $\begin{array}{l}86 \\
(60,1 \%)\end{array}$ & $M=13,13, d p=2,20$ \\
\hline Usava cola, solventes & $\begin{array}{l}20 \\
(20,2 \%)\end{array}$ & $\begin{array}{l}2 \\
(13,3 \%)\end{array}$ & $\begin{array}{l}22 \\
(15,4 \%)\end{array}$ & $M=14,23, d p=2,20$ \\
\hline Usava crack & $\begin{array}{l}19 \\
(19 \%)\end{array}$ & $\begin{array}{l}6 \\
(40 \%)\end{array}$ & $\begin{array}{l}25 \\
(17,5 \%)\end{array}$ & $M=14,83, d p=1,85$ \\
\hline Usava maconha* & $\begin{array}{l}76 \\
(73,8 \%)\end{array}$ & $\begin{array}{l}5 \\
(33,3 \%)\end{array}$ & $\begin{array}{l}81 \\
(56,6 \%)\end{array}$ & $M=13,82, d p=1,82$ \\
\hline Usava cocaína** & $\begin{array}{l}62 \\
(59,6 \%)\end{array}$ & $\begin{array}{l}4 \\
(28,6 \%)\end{array}$ & $\begin{array}{l}66 \\
(46,2 \%)\end{array}$ & $M=15, d p=1,5$ \\
\hline
\end{tabular}

Nota: ${ }^{*}{ }^{2}(1)=9,95, \mathrm{p}=0,002 ;{ }^{* *} \mathrm{X} 2(1)=4,82, \mathrm{p}=0,02$

Fonte: Dados da pesquisa.

De acordo com a tabela 2, a maioria dos adolescentes tem algum familiar e algum amigo usuário de drogas. Ressalta-se o elevado percentual de amigos usuários $(88,8 \%)$, pois, conforme apontam alguns autores, o uso de drogas na adolescência pode estar associado à necessidade de se incorporar ao grupo de amigos (Nardi, 2010; Vasters \& Pillon, 2011; Wagner \& Oliveira, 2007). Anderson (1999) mostrou, em estudo com 
adolescentes infratores, que o uso de drogas acontecia principalmente devido à pressão feita pelo grupo de pares. De acordo com o autor, "drogas e gangs andam juntas".

A média de idade para o início do uso de substâncias lícitas, álcool e cigarro foi de 13 anos. O I Levantamento sobre os Padróes de Consumo de Álcool na População Brasileira, realizado pela Secretaria Nacional de Políticas sobre Drogas [SENAD], também revelou que o início do hábito de beber ocorre em média aos 13 anos de idade (Laranjeira, Pinsky \& Caetano, 2007), assim como o estudo realizado com adolescentes em conflito com a lei no Sul do Brasil, o qual revelou que a maioria dos usuários iniciou o consumo abusivo entre 13 e 15 anos (Davoglio \& Gauer, 2011).

As drogas mais utilizadas pelos adolescentes participantes deste estudo foram o álcool e o cigarro, resultados também já apontados anteriormente (Schenker \& Minayo, 2005). Porém, mais uma vez, o uso de uma droga ilícita, a maconha, está com percentual quase emparelhado ao das drogas lícitas entre os adolescentes participantes deste estudo, o que demonstra a exposição, cada vez mais cedo, desses jovens a fatores de risco. Entre as drogas ilícitas, a mais utilizada é a maconha, seguida da cocaína e do crack. Relatório sobre essa população no Brasil apontou que 85,6\% dos jovens já eram usuários de drogas antes da internação. As drogas mais citadas foram maconha $(67,1 \%)$, álcool $(32,4 \%)$, cocaína e $\operatorname{crack}(31,3 \%)$, $\mathrm{e}$ inalantes (22,6\%) (ANCED/FÓRUM DCA, 2004). Observou-se diferença significativa entre os sexos para o uso de maconha e cocaína, com percentuais mais altos para os meninos. Porém não é o que indica a Secretaria de Direitos Humanos [SDH] (2010), a qual assinala que o uso de álcool, cigarro e maconha são iguais para homens e mulheres. No entanto é assinalado também que, entre as gangues, as meninas dividemse entre usuárias e não usuárias de substâncias, ao passo que a grande maioria dos garotos afirma-se usuária, indicando o maior número de adolescentes do sexo masculino que fazem uso de drogas. Um estudo realizado com 27990 alunos de ensino fundamental e médio, de 10 a 19 anos, revelou que não houve diferença significativa no consumo de drogas entre meninas e meninos (Tavares, Beria \& Lima, 2004). Esses dados sugerem que as diferenças encontradas nessa amostra podem estar refletindo uma característica específica desses participantes.

Conforme a tabela 3, mais da metade dos adolescentes vivenciou experiências estressoras em sua vida, como alguém da casa estar desempregado, um familiar estar ou ter estado preso e a morte de 
alguém importante. $\mathrm{O}$ desemprego e a prisão de um membro familiar colaboram para o decréscimo da renda e o aumento da pobreza. Além disso, Patterson, Reid e Dishion (1992) já apontavam que a presença de comportamento antissocial em algum membro familiar pode atuar como importante fator preditor desse mesmo comportamento em outros membros, o que aparece também em estudos mais recentes (Nardi, 2010; Pacheco \& Hutz, 2009). Portanto, percebe-se a exposição dos participantes não só a fatores de risco dentro da própria família como também a modelos de comportamento antissocial no ambiente familiar.

$\mathrm{O}$ evento estressor com maior percentual entre os participantes foi a morte de alguém importante. Deve-se considerar que qualquer perda é acompanhada por perdas secundárias, pois uma morte modifica o contexto no qual o indivíduo está inserido, trazendo outras consequências no seu cotidiano (Domingos \& Maluf, 2003). Um estudo que examinou experiências de perda e luto de escolares adolescentes revelou que, dependendo da forma como o jovem lida com a morte, diversos comportamentos e sentimentos podem ser desencadeados, tais como um baixo desempenho escolar, intensa desorientação, raiva, hostilidade, desespero e perda de controle (Domingos \& Maluf, 2003). Outro estudo, que investigou a frequência e o impacto de eventos de vida estressores em 330 estudantes de 12 a 17 anos, revelou que a morte de algum familiar foi um evento de grande ocorrência entre os participantes e que situaçôes relacionadas à perda de membros da família foram consideradas pelos adolescentes com elevado impacto (Kristensen, Leon, D'Incao \& Dell'Aglio, 2004).

Tabela 3 - Ocorrência de eventos estressores e suicídio

\begin{tabular}{l|l|l|l|l}
\hline \multirow{2}{*}{} & Variável & \multicolumn{3}{|c}{$f$ e $\%$} \\
\cline { 3 - 5 } & & Masc. & Fem. & Total \\
\hline \multirow{2}{*}{$\begin{array}{l}\text { Eventos } \\
\text { estressores }\end{array}$} & Alguém da casa está & 66 & 7 & 73 \\
& desempregado & $(54,5 \%)$ & $(58,3 \%)$ & $(51 \%)$ \\
& Alguém da família está ou & 74 & 7 & 81 \\
& esteve preso & $(61,7 \%)$ & $(58,3 \%)$ & $(56,6 \%)$ \\
& Alguém muito importante & 95 & 12 & 107 \\
& faleceu & $(79,2 \%)$ & $(92,3 \%)$ & $(74,8 \%)$ \\
& Puicídio & 19 & 8 & 27 \\
& Pensou em se matar* & $(16,1 \%)$ & $(61,5 \%)$ & $(18,9 \%)$ \\
& Tentou se matar** & 14 & 6 & 20 \\
& & $(15,1 \%)$ & $(46,2 \%)$ & $(14 \%)$ \\
\hline
\end{tabular}

Nota: ${ }^{*} \mathrm{X}^{2}(1)=14,77, \mathrm{p}<0,001 ;{ }^{* *} \mathrm{X}^{2}(1)=7,2, \mathrm{p}=0,007$. 
Fonte: Dados da pesquisa.

De acordo com Móscicki (1997), a perda de alguém que dá suporte emocional ou material pode contribuir para futuras tentativas de suicídio. Outros autores apoiam essa ideia, mencionando que a perda de pessoas significativas aumenta consideravelmente a probabilidade de suicídio (Toro, Paniagua, González \& Montoya, 2009). Neste estudo, 18,9\% dos adolescentes já pensaram em se matar e 14\% já tentaram se matar. Esses dados são similares aos resultados da pesquisa de Morris et al. (1995), realizado com 1801 adolescentes autores de ato infracional nos Estados Unidos. Essa pesquisa indicou que 20\% dos participantes já haviam planejado uma tentativa de suicídio, enquanto que $16 \%$ já haviam tentado o suicídio.

Móscicki (1997) aponta que as taxas de suicídio variam de acordo com idade, cor, nível socioeconômico, estado civil e gênero, sendo mais frequente no sexo feminino. Os resultados deste estudo vão ao encontro desse dado, revelando que as meninas apresentaram um percentual mais elevado do que os meninos nos itens ideação e tentativa de suicídio. $\mathrm{O}$ maior número de tentativas entre adolescentes do sexo feminino pode estar relacionado, segundo Bahls e Bahls (2002), ao maior índice de depressão dessa população, já que a literatura aponta que a depressão desempenha um importante papel no comportamento suicida.

Estudos realizados com jovens da mesma faixa etária também mostraram percentuais de tentativa de suicídio maiores entre adolescentes do sexo feminino, numa proporção de três para um (Abasse, Coimbra, Silva \& Souza, 2009; Avanci, Pedrão \& Costa Júnior, 2005). Porém não é só o gênero que é considerado um fator de risco à tentativa de suicídio. Diversos autores assinalam a vivência de outros eventos estressores, como o desemprego, a prisão e o uso de drogas como aspectos relacionados ao suicídio (Braga, 2011; Móscicki, 1997). Portanto se percebe que os adolescentes em conflito com a lei participantes deste estudo apresentam os fatores de risco relacionados por muitos autores à questão do suicídio, demonstrando a vulnerabilidade à qual estão expostos.

A tabela 4 apresenta a média de expectativas de futuro dos participantes. Com base nos dados, observam-se maiores expectativas quanto a ter uma família, ser respeitado na comunidade, ser saudável, ter casa própria e amigos que darão apoio. Por outro lado, os participantes apresentaram uma percepção mais negativa no que se refere a entrar na universidade ou mesmo concluir o ensino médio. 
Tabela 4 - Médias e desvios-padrão das expectativas de futuro

\begin{tabular}{lll}
\hline Plano & M & dp \\
\hline Concluir o ensino médio & 3,38 & 1,4 \\
Entrar na universidade & 2,64 & 1,37 \\
Ter emprego que garanta boa qualidade de vida & 3,67 & 1,27 \\
Ter casa própria & 4,17 & 1,22 \\
Ter trabalho que dará satisfação & 3,90 & 1,17 \\
Ter uma família & 4,50 & 0,97 \\
Ser saudável a maior parte do tempo & 4,37 & 0,99 \\
Ser respeitado na comunidade & 4,45 & 0,92 \\
Ter amigos que darão apoio & 4,05 & 1,18 \\
\hline
\end{tabular}

Fonte: Dados da pesquisa.

Sobre a expectativa de "ter uma família", apontada neste estudo como a mais almejada, Gonçalves et al. (2008) apontam que a maioria dos jovens participantes da sua pesquisa sobre problemas e enfrentamentos na juventude anunciou o desejo de constituir família. Com esse projeto, os jovens esperam lograr qualidades que pais e mães podem ter (pais esforçados, mães dedicadas, entre outros) e a superação de adversidades presentes em suas próprias famílias, com uma expectativa de ter uma família no futuro baseada na família atual, embora de maneira idealizada. Relacionado a isso, pode-se pensar que as altas expectativas de ter uma casa própria, demonstrem a busca de consolidação do projeto de vida de ter uma família, que tem na casa a sua concretização (Gonçalves et al., 2008).

Quanto a ser respeitado na comunidade, como aponta Steinberg (1999), a adolescência consiste na gradual assunção de papéis e funções que conferem um status adulto. Além disso, para que esse status se consolide, é necessária a aceitação dos outros adultos. Portanto ser respeitado na comunidade pode ser reflexo desse movimento natural na adolescência, ilustrados, por exemplo, no discurso de adolescentes do sexo feminino (Secretaria de Direitos Humanos, 2010 \& Pantoja, 2003), para quem a maternidade é uma forma de atingir esse objetivo.

Ser saudável a maior parte do tempo também apresentou uma média mais alta de expectativas. De acordo com Paredes e Pecora (2004), a conquista da saúde está relacionada ao sentir-se bem, o que influencia a realização social e pessoal dos adolescentes. Porém, embora os participantes apresentem 
expectativas positivas quanto a ser saudável, muitas vezes manifestam comportamentos contrários, tais como o uso de drogas. Dessa forma, observa-se que os adolescentes nem sempre apresentam um comportamento direcionado ao alcance de seus objetivos. Por exemplo, este e outros estudos (Gallo \& Williams, 2005; Oliveira \& Assis, 1999) apontam altos índices de reprovação e evasão escolar entre adolescentes em conflito com a lei, embora eles tenham desejo de concluir o ensino médio ou entrar na universidade.

Já a expectativa dos participantes em ter amigos que darão apoio está em conformidade com o que alguns autores apontam sobre a importância do grupo de pares na adolescência (Dodge \& Gonzales, 2009; Monahan, Steinberg $\&$ Cauffman, 2009). De acordo com Dodge e Gonzales (2009), o grupo de pares, juntamente com a família, é um dos contextos mais importantes de socialização. Todavia o grupo de pares pode ser um fator de risco ao ato infracional, e muitas infrações podem ser cometidas nessa relação (Hein, 2004), assim como o uso de drogas ilícitas. Gonçalves et al. (2008) ressaltam que os amigos são entendidos pelos adolescentes como soluções quando em consonância com os seus projetos, e problemas quando se tornam obstáculo para a consecução de seus objetivos.

Quanto às expectativas com médias mais baixas, entrar na universidade e concluir o ensino médio, ressalta-se a dificuldade que muitos desses adolescentes têm em relação aos estudos, fato apontado por muitos autores (Gallo \& Williams, 2005; Hein, 2004) e observado por meio dos índices de repetência e expulsão da escola aqui encontrados. Embora os estudos possam ser entendidos como meios de ascensão social e garantia de uma profissão por adolescentes de escolas públicas (Paredes \& Pecora, 2004), os adolescentes em conflito com a lei podem se afastar dos estudos justamente em função do trabalho, dado que as urgências de suas vidas lhes cobram dedicação ao trabalho e ao seu sustento (e, por vezes, ao de suas famílias) o que compromete os seus planos acadêmicos (Jacobina \& Costa, 2007). Por outro lado, o discurso de promoção social por vias ilegais, como o tráfico de drogas, pode contribuir para que haja um descrédito da escola como meio de qualificação profissional (Paredes \& Pecora, 2004). Esse discurso pode ser percebido em estudo realizado pela Secretaria de Direitos Humanos com adolescentes pertencentes a gangues (Secretaria de Direitos Humanos, 2010), pois muitos jovens mencionaram a escola como um espaço de convivência social e não como um projeto de vida em si ou como um meio de atingir projetos de vida. Além disso, em relação ao acesso ao ensino superior, Oliveira, Pinto e Souza (2003) apontam que esse ainda se mantém bastante elitizado, sendo que as camadas populares recebem menos condições de competir por vagas no 
sistema público de ensino, dificultando o acesso desses jovens à universidade.

\section{Considerações finais}

Com base nos resultados apresentados e discutidos, percebe-se a importância de considerar todo o contexto que envolve um adolescente em conflito com lei, tendo em vista que foi observada a presença de inúmeros fatores de risco, principalmente no contexto familiar. Portanto, torna-se extremamente importante um trabalho mais amplo com esses adolescentes, que envolva a família, a comunidade e os profissionais próximos a essa comunidade, constituindo uma rede de proteção, que forneça apoio, limite e afeto, em contraposição às vivências de violência e risco a que estão expostos.

Entre os diversos aspectos que se constituem em risco para esses adolescentes, ressalta-se a questão do uso de drogas lícitas e ilícitas, necessitando de maior atenção por parte de profissionais e políticas públicas para os adolescentes em conflito com a lei. Deve-se considerar o risco do início precoce do uso de drogas, principalmente tendo em vista que o desenvolvimento cognitivo na infância ainda não permite uma avaliação mais clara quanto às consequências de tal comportamento (Priuli \& Moraes, 2007). Quanto aos eventos estressores, os identificados entre os participantes têm potencial de impacto negativo, como a prisão de algum familiar ou morte de alguém importante, sendo diferentes daqueles apontados por alguns autores (Oliveira \& Costa, 1997), como característicos da fase da adolescência. Esses dados podem levar à reflexão de que muitas vezes os adolescentes em conflito com a lei não vivenciam um desenvolvimento visto como próprio da adolescência, pois são surpreendidos com uma ampla variedade de fatores de risco ao longo de suas vidas com os quais precisam aprender a lidar. O estudo de Espíndula e Santos (2004) apresenta a possibilidade de duas representações de adolescência: a "normal", caracterizada por uma fase de transição, onipotência e dificuldades no processo de desenvolvimento; e a infratora, dita como "diferente", oriunda de uma "família desestruturada", a qual serve de modelo para as interaçôes conflituosas que levam à infração. De acordo com Sapienza e Pedromônico (2005), quando muitas situaçóes de risco se associam, elas dificultam o cumprimento da agenda desenvolvimental "normal", assim como a aquisição de habilidades e o desempenho de papéis sociais adequados.

O fenômeno da violência tem provocado um cenário de crescente preocupação na sociedade, que leva à reflexão sobre a responsabilização dos jovens pelo crescimento da criminalidade e, consequentemente, à exigência de penalização ao jovem autor de ato infracional, gerando impactos nas discussões sobre a redução da maioridade penal (Almeida \& Sousa, 2010). Porém cabe refletir sobre essa responsabilização do adolescente, tendo em vista os contextos em que este está inserido e se desenvolve. 
Neste estudo, foram destacados alguns fatores de risco aos quais esses adolescentes estão expostos. De acordo com Feijó e Assis (2004), esses fatores estão associados a um contexto de exclusão social em que vivem as famílias dos jovens infratores. $\mathrm{Na}$ ideia de exclusão, inserem-se várias formas de discriminação, levando a um conjunto de vulnerabilidades difíceis de superar. Corroborando essa ideia, Estevam, Coutinho e Araújo (2009) mencionam que, geralmente, antes de conhecerem a criminalidade, esses adolescentes sobreviviam em situações de extrema carência afetiva, educacional e material. Deperon e Pinho (2012) refletem sobre os diversos direitos que foram negados a um jovem em conflito com a lei ao longo de seu desenvolvimento. $\mathrm{O}$ ECA (1990) aponta os direitos desses jovens, tais como o direito à liberdade, ao respeito e à dignidade, assim como o direito à convivência familiar e comunitária. Há, portanto, uma clara violação de quase todos esses direitos no momento em que um jovem é privado de sua liberdade.

O Sistema Nacional de Atendimento Socioeducativo [SINASE] (2012) (Lei Federal n. 12.594/2012) corrobora alguns aspectos do ECA, ressaltando, em seu artigo 49, inciso III, o direito do adolescentes infrator de ser respeitado em sua personalidade, intimidade, liberdade de pensamento e religião, e em todos os direitos não expressamente limitados na sentença. Estudos como o de Estevam, Coutinho, \& Araújo (2009) revelam que as condições de adolescentes dentro das instituições de privação de liberdade contribuem para a criação de uma imagem de ser humano inferior e ameaça à sociedade, contrariando, assim, direitos previstos tanto no ECA (1990) como no SINASE (2012). Portanto, percebe-se a urgência de investimento em políticas públicas voltadas aos adolescentes e às suas famílias, com o objetivo de atuar preventivamente junto a essa população, buscando reduzir o número de jovens envolvidos na criminalidade e colocando em prática as leis e direitos vigentes.

\section{Referências}

Abasse, M. L. F., Coimbra, R., Silva, T. C., \& Souza, E. R. (2009). Análise epidemiológica da morbimortalidade por suicídio entre adolescentes em Minas Gerais, Brasil. Ciência e Saúde Coletiva, 14 (2), 407-416.

Adorno, S. (2010). A violência na sociedade brasileira. Juventudee delinquência como problemas sociais. Revista Brasileira Adolescência e Conflitualidade, 2 (2), $1-11$.

Almeida, R. O., \& Sousa, L. E. E. M. (2010). Liberdade assistida: socioeducação e punição em conflito. O público e o privado, 15, 189-210. 
Anderson, N. L. R. (1999). Perceptions about substance use among male adolescents in juvenile detention. Western Journal of Nursing Research, 21 (5), 652-672.

Associação Nacional dos Centros de Defesa da Criança e do Adolescente \& Fórum Nacional Permanente das Entidades Não Governamentais de Defesa dos Direitos da Criança e do Adolescente (2004). Relatório sobre a situação dos direitos da criança e do adolescente no Brasil. Recuperado em 10 janeiro, 2012, de http://pfdc.pgr.mpf.mp.br/atuacao-e-conteudos-de-apoio/publicacoes/ crianca-e-adolescente/relatorio_situacao_direitos_crianca_adolescente_ Brasil.

Anderson, N. L. R. (1999). Perceptions about substance use among male adolescents in juvenile detention. Western Journal of Nursing Research, 21 (5), 652-672.

Avanci, R. C., Pedrão, L. J., \& Costa Júnior, M. L. (2005). Perfil do adolescente que tenta suicídio admitido em uma unidade de emergência. Revista Brasileira de Enfermagem, 58 (5), 535-539.

Bahls, S., \& Bahls, F. R. C. (2002). Depressão na adolescência: características clínicas. Interação em Psicologia, 6 (1), 49-57.

Braga, L. L. (2011). Exposição à violência: impacto em adolescentes de diferentes contextos. Dissertação de Mestrado, Universidade Federal do Rio Grande do Sul, Porto Alegre, RS, Brasil.

Branco, B. M., Wagner, A., \& Demarchi, K. A. (2008). Adolescentes infratores: rede social e funcionamento familiar. Psicologia: Reflexão e Crítica, 21 (1), 125-132.

Costa, C. R. B. S. F., \& Assis, S. G. (2006). Fatores protetivos a adolescentes em conflito com a lei no contexto socioeducativo. Psicologia \& Sociedade, 18 (3), 74-81.

Davoglio, T. R., \& Gauer, G. J. C. (2011). Adolescentes em conflito com a lei: aspectos sociodemográficos de uma amostra em medida socioeducativa com privação de liberdade. Contextos Clínicos, 4 (1), 42-52.

Dell'Aglio, D. D., \& Koller, S. H., Cerqueira-Santos, E., \& Colaço, V. F. R. (2011). Revisando o questionário da juventude brasileira: uma nova proposta. In D. D. Dell'Aglio \& S. H. Koller (Ed.). Adolescência e juventude: vulnerabilidade e contextos de proteção. (pp. 259-270). São Paulo: Casa do Psicólogo.

Dell'Aglio, D. D., Santos, S. S., \& Borges, J. L. (2004). Infração juvenil feminina: uma trajetória de abandonos. Interação em Psicologia, 8 (2), 191- 
198.

Deperon, R., \& Pinho, C. C. M. (2012). Adolescente em conflito com a lei: vítima e vitimizador. Psicologia Argumento, 30 (70), 441-451.

Dias, A. C. G., Arpini, D. M., \& Simon, B. R. (2011). Um olhar sobre a família de jovens que cumprem medidas socioeducativas. Psicologia \& Sociedade, 23 (3), 526-535.

Dodge K., \& Gonzales, N. (2009). Family and peer influences on adolescent behavior and risk-taking. Paper presented at IOM Committee on the Science of Adolescence Workshop, Washington, DC. Recuperado em 10 de janeiro, 2012, de http://www.iom.edu/ /media/Files/Activity\%20Files/Children/ AdolescenceWS/Commissioned\%20Papers/dodge_gonzales_paper.pdf.

Domingos, B., \& Maluf, M. R. (2003). Experiências de perda e de luto em escolares de 13 a 18 anos. Psicologia: Reflexão e Crítica, 16 (3), 577-589.

Eizirik, M., \& Bergmann, D. S. (2004). Ausência paterna e sua repercussão no desenvolvimento da criança e do adolescente: um relato de caso. Revista de Psiquiatria do Rio Grande do Sul, 26 (3), 330-336.

Espíndula, D. H. P., \& Santos, M. F. S. (2004). Representações sobre a adolescência a partir da ótica dos educadores sociais de adolescentes em conflito com a lei. Psicologia em Estudo (Maringá), 9 (3), 357-367.

Estevam, I. D., Coutinho, M. P. L., \& Araújo, L. F. (2009). Os desafios da prática socioeducativa de privação de liberdade em adolescentes em conflito com a lei: ressocialização ou exclusão social? Psico, 40 (1), 64-72.

Feijó, M. C., \&Assis, S. G. de. (2004). O contextode exclusãosocial edevulnerabilidade de jovens infratores e de suas famílias. Estudos de Psicologia, 9 (1), 157-166.

Ferigolo, M., Barbosa, F. S., Arbo, E., Malysz, A. S., Stein, A. T., Barros, H. M. T. (2004). Prevalência do consumo de drogas na FEBEM, Porto Alegre. Revista Brasileira de Psiquiatria, 26 (1), 10-16.

Fukuda, C. C., Brasil, K. T., \& Alves, P. B. (2009). Fatores de risco e proteção: consideraçōes sobre gênero. In R. M. C. Libório \& S. H. Koller (Ed.). Adolescência e juventude: risco e proteção na realidade brasileira. (pp. 107-131). São Paulo: Casa do Psicólogo.

Furlani, D. D., \& Bomfim, Z. A. C. (2010). Juventude e afetividade: tecendo projetos de vida pela construção dos mapas afetivos. Psicologia \& Sociedade, $22(1), 50-59$. 
Gallo, A. E., \& Williams, L. C. A. (2005). Adolescentes em conflito com a lei: uma revisão dos fatores de risco para a conduta infracional. Psicologia: Teoria e Prática, 7 (1), 81-95.

Garbarino, J. (2009). Why are adolescents violent? Ciência e Saúde Coletiva, 14 (2), 533-538.

Gonçalves, H. S., Borsoi, T. S., Santiago, M. A., Lino, M. V., Lima, I. N., \& Federico, R. G. (2008). Problemas da juventude e seus enfrentamentos: um estudo de representações sociais. Psicologia \& Sociedade, 20 (2), 217-225.

Gröer, M. W., Thomas, S. P., \& Schoffner, D. (1992). Adolescence stress and coping: a longitudinal study. Research in Nursing and Health, 15 (3), 209-217.

Günther, I. A., \& Günther, H. (1998). Brasílias pobres, Brasílias ricas: perspectivas de futuro entre adolescentes. Psicologia: Reflexão e Crítica, 11, 191-207.

Hein, A. (2004). Factores de riesgo y deincuencia juvenil: revisión de la literatura nacional e internacional. Santiago: Fundación Paz e Ciudadana. Recuperado em 4 de setembro, 2010, de http://www.pazciudadana.cl/docs/ pub_200906231 90509.pdf.

Instituto Brasileiro de Geografia e Estatística. (2010). Resultados preliminares do universo do censo demográfico. Rio de Janeiro: IBGE. Recuperado em 9 de setembro, 2011, de http://www.ibge.gov.br/home/presidencia/noticias/ imprensa/ppts/0000000408. pdfJacobina, O. M. P., \& Costa, L. F. (2007). "Para não ser bandido": trabalho e adolescentes em conflito com a lei. Cadernos de Psicologia Social e do Trabalho, 10 (2), 95-110.

Kristensen, C. H., Leon, J. S., D’Incao, D. B., \& Dell'Aglio, D. D. (2004). Análise da frequência e do impacto de eventos estressores em uma amostra de adolescentes. Interação em Psicologia, 8 (1), 45-55.

Laranjeira, R., Pinsky, I., \& Caetano, R. (2007). I Levantamento nacional sobre os padrões de consumo de álcool na população brasileira. Brasília: Secretaria Nacional Antidrogas - Senad.

Lei Federal n. 8.069, de 13 de julho de 1990. (1990, 13 de julho). Dispõe sobre o Estatuto da Criança e do Adolescente e dá outras providências. Recuperado em 27 de setembro, 2013, de http://www.planalto.gov.br/ccivil_03/Leis/ L8069.htm. 
Lei Federal n. 12.594, de 18 de janeiro de 2012. (2012, 18 de janeiro). Institui o Sistema Nacional de Atendimento Socioeducativo (Sinase), regulamenta a execução das medidas socioeducativas destinadas a adolescente que pratique ato infracional; e altera as Leis n. 8.069, de 13 de julho de 1990 (Estatuto da Criança e do Adolescente); 7.560, de 19 de dezembro de 1986, 7.998, de 11 de janeiro de 1990, 5.537, de 21 de novembro de 1968, 8.315, de 23 de dezembro de 1991, 8.706, de 14 de setembro de 1993, os DecretosLeis nos 4.048, de 22 de janeiro de 1942, 8.621, de 10 de janeiro de 1946, e a Consolidação das Leis do Trabalho (CLT), aprovada pelo Decreto-Lei no 5.452, de $1^{\circ}$ de maio de 1943. Recuperado em 27 de setembro, 2013, de http://www.planalto.gov.br/CCIVIL_03/_Ato2011-2014/2012/Lei/ L12594.htm.

Lodewijks, H. P. B., Ruiter, C., \& Doreleijers, T. A. H. (2010). The impact of protective factors in desistance from violent reoffending: a study in three samples of adolescent offenders. Journal of Interpersonal Violence, 25 (3), 568587.

Malvasi, P. A. (2011). Entre a frieza, o cálculo e a "vida loka": violência e sofrimento no trajeto de um adolescente em cumprimento de medida socioeducativa. Saúde e Sociedade, 20 (1), 156-170.

Maruschi, M. C., Estevão, R., \& Bazon, M. R. (2012). Risco de persistência na conduta infracional. Estudos de Psicologia, 29 (supl.), 679-687.

Monahan, K. C., Steinberg, L., \& Cauffman, E. (2009). Affiliation with antisocial peers, susceptibility to peer influence, and antisocial behavior during the transition to adulthood. Development Psychology, 45 (6), 1520-1530.

Morris, R. E., Harrison, E. A., Knox, G. W., Tromanhauser, E., Marquis, D. K., \& Watts, L. L. (1995). Health risk behavioral survey from 39 juvenile correctional facilities in the United States. Journal of Adolescent Health, 17, 334-344.

Móscicki, E. K. (1997). Identification of suicide risk factors using epidemiologic studies. Psychiatric Clinics of North America, 20 (3), 499-517.

Muller, F. Barboza, P. S., Oliveira, C. C., Santos, R. R. G. \& Paludo, S. S. (2009). Perspectivas de adolescentes em conflito com a lei sobre o delito, a medida de internação e as expectativas futuras. Revista Brasileira Adolescência e Conflitualidade, 1 (1), 70-87. 
Nardi, F. L. (2010). Adolescentes em conflito com a lei: percepçôes sobre família, ato infracional e medida socioeducativa. Dissertação de Mestrado, Universidade Federal do Rio Grande do Sul, Porto Alegre, RS, Brasil.

Nardi, F. L., Cunha, S. M., Bizarro, L. \& Dell'Aglio, D. D. (2012). Drug use and antisocial behavior among adolescents attending public schools in Brazil. Trends Psychiatry Psychother, 34 (2), 80-86.

Nascimento, I. P. (2006). Projeto de vida de adolescentes do ensino médio: um estudo psicossocial sobre suas representações. Imaginário, 12 (12), 55-80.

Oliveira, C. A., \& Costa, A. E. (1997). Categorias de conflitos no cotidiano de adolescentes mineiros. Psicologia Reflexão e Crítica, 10 (1), 87-104.

Oliveira, M. B., \& Assis, S. G. (1999). Os adolescentes infratores do Rio de Janeiro e as instituições que os "ressocializam": a perpetuação do descaso. Cadernos de Saúde Pública, 15 (4), 831-844.

Oliveira, M. C. S. L., Pinto, R. G., \& Souza, A. S. (2003). Perspectivas de futuro entre adolescentes: universidade, trabalho e relacionamentos na transição para a vida adulta. Temas em Psicologia, 11, 16-27.

Pacheco, J. T. B. \& Hutz, C. (2009). Variáveis familiares preditoras do comportamento anti-social em adolescentes autores de atos infracionais. Psicologia: Teoria e Pesquisa, 25 (2), 213-219.

Pantoja, A. L. N. (2003). "Ser alguém na vida": uma análise socioantropológica da gravidez/maternidade na adolescência, em Belém do Pará, Brasil. Caderno de Saúde Pública, 19 (2), 335-343.

Paredes, E. C., \& Pecora, A. R. (2004). Questionando o futuro: as representações sociais de jovens estudantes. Psicologia: Teoria e Prática, 6 (3), 49-65.

Patterson, G., Reid, J. \& Dishion, T. (1992). Antisocial boys. [s.l.]: Castalia Publishing Company.

Pereira, S. E. F. N., \& Sudbrack, M. F. O. (2008). Drogadição e atos infracionais na voz do adolescente em conflito com a lei. Psicologia: Teoria e Pesquisa, 24 (2), 151-159.

Priuli, R. M. A., \& Moraes, M. S. (2007). Adolescentes em conflito com a lei. Ciência \& Saúde Coletiva, 12 (5), 1185-1192. 
Rigoni, M., Oliveira, M., Moraes, J., \& Zambom, L. F. (2007). O consumo de maconha na adolescência e o prejuízo nas funções cognitivas. Psicologia em Estudo, 12 (2), 267-275.

Sapienza, G., \& Pedromônico, M. R. M. (2005). Risco, proteção e resiliência no desenvolvimento da criança e do adolescente. Psicologia em Estudo, 10 (2), 209-216.

Secretaria de Direitos Humanos. (2010). Gangues, gênero e juventudes: donas de rocha e sujeitos cabulosos. Brasília: SDH.

Schenker, M., \& Minayo, M. C. S. (2005). Fatores de risco e de proteção para o uso de drogas na adolescência. Ciência \& Saúde Coletiva, 10, 707-717.

Senna, S. R. C. M., \& Dessen, M. A. (2012). Contribuições das teorias do desenvolvimento humano para a concepção contemporânea da adolescência. Psicologia: Teoria e Pesquisa, 28 (1), 101-108.

Steinberg, L., (1999). Adolescence (5th ed.). Boston: McGraw-Hill College.

Tavares, B. F., Beria, J. U., \& Lima, M. S. (2004). Fatores associados ao uso de drogas entre adolescentes escolares. Revista de Saúde Pública, 38 (6), 787-796.

Toro, D. C., Paniagua, R. E., González, C. M. \& Montoya, B. (2009). Caracterización de adolescentes escolarizados con riesgo de suicidio, Medellín, 2006. Revista da Facultad Nacional de Salud Pública, 27 (3), 302-308.

Vasters, G. P. \& Pillon, S. C., (2011). O uso de drogas por adolescentes e suas percepções sobre adesão e abandono de tratamento especializado. Revista Latino-Americana de Enfermagem, 19 (2). Recuperado em 27 de setembro, 2013, de http://www.scielo.br/pdf/rlae/v19n2/pt_13.pdf.

Vermeiren, R. (2003). Psychopathology and delinquency in adolescents: a descriptive and developmental perspective. Clinical Psychology Review, 23, 277-318.

Wagner, M. \& Oliveira, M. (2007). Habilidades sociais e abuso de drogas em adolescentes. Psicologia Clínica, 19 (2), 101-116.

Zamora, M. H. (2008). Adolescentes em conflito com a lei: um breve exame da produção recente em psicologia. Revista Eletrônica Polêmica. Recuperado em 26 setembro, 2013, de http://www.sinddegase.org.br/site/ pdf/estudos/08zamor_psicologia.pdf. 